\title{
Impaired executive functions in experimental model of temporal lobe epilepsy
}

\author{
Prejuízo das funções executivas no modelo experimental de epilepsia do lobo temporal \\ Fabiane Ochai Ramos ${ }^{1,2}$, Luiz Renato Rodrigues Carreiro', Fulvio Alexandre Scorza ${ }^{2}$, \\ Roberta Monterazzo Cysneiros ${ }^{1}$
}

\begin{abstract}
Objective: The present study aimed to investigate cognitive and behavioural changes consistent with attention deficit hyperactivity disorder (ADHD - like behavior in male Wistar rats with temporal lobe epilepsy (TLE). Method: Male Wistar rats at 25 day of age were submitted to animal model of TLE by pilocarpine injection ( $350 \mathrm{mg} / \mathrm{kg}$, ip) and a control group received saline $0.9 \%$. The animals were continuously video monitored up to the end of experiments. The behavioural tests (open field, elevated plus maze and operant conditioning box) started from 60 days postnatal. Results: Animals with TLE exhibited elevated locomotor activity, reduced level of anxiety-related behavior, impulsivity and impaired visuospatial working memory. Conclusion: Taken as a whole, we concluded that animals with TLE exhibited some cognitive and behavioural changes consistent with ADHD-like behavior.
\end{abstract}

Keywords: Epilepsy, temporal lobe; executive function; pilocarpine; memory, short-term.

\section{RESUMO}

Objetivo: O presente estudo teve como objetivo investigar as alterações cognitivas e comportamentais consistentes com o comportament de transtorno de deficit de atenção e hiperatividade (TDAH) -like em ratos Wistar machos com epilepsia do lobo temporal (ELT). Método: Ratos Wistar machos com 25 dias de vida foram submetidos a modelo animal de ELT pela injeção de pilocarpina (350 mg / kg, ip) e grupo controle recebeu salina 0,9\%. Os animais foram monitorados continuamente por vídeo até ao final dos experimentos. Os testes comportamentais (campo aberto, labirinto em cruz elevado e caixa de condicionamento operante) começaram a partir de 60 dias pós-natal. Resultados: Os animais com ELT exibiram aumento da atividade locomotora, redução do comportamento relacionado com a ansiedade, impulsividade e prejuízo da memória de trabalho visuospatial. Conclusão: Em conjunto, concluímos que os animais com ELT apresentaram algumas alterações cognitivas e comportamentais consistentes com o comportamento TDAH-like.

Palavras-chave: epilepsia do lobo temporal; função executiva; pilocarpina; memória de curto prazo.

WITH approximately 65 million people affected worldwide, epilepsy is one of the most common, chronic and serious neurological disease $e^{1,2,3,4}$. Temporal lobe epilepsy (TLE) is the commonest form of human epilepsy, affecting approximately $40 \%$ of patients and often resistant to antiepileptic drugs ${ }^{5,6,7}$. From the structural and functional points of view, TLE is often associated with specific structural abnormalities that affect limbic structures as well as frontal lobe, which is associated with cognitive processes, including executive functions, working memory (WM), decision making, planning, cognitive flexibility and sustained attention ${ }^{8,9}$. Epilepsy is frequently associated with some psychiatry comorbities, such as attention deficit hyperactivity disorder (ADHD) and autism spectrum disorder (ASD). The prevalence of ADHD in children with epilepsy is higher than in general population, being estimated in $30-40 \%^{10,11,12}$. Although there is a well-established relationship between the two disorders, the underlying mechanisms are still unclear and more research needs to be performed. Thus, the animal models allow investigate many issues related to epilepsy in the absence of iatrogenic neurobehavioral abnormalities. Based on these, the present study aimed to investigate ADHD-like behaviour in male rats with pilocarpine-induced TLE.

\footnotetext{
'Universidade Presbiteriana Mackenzie, Laboratório de Neurobiologia, Programa de Pós-Graduação em Distúrbios do Desenvolvimento, São Paulo SP, Brasil; ${ }^{2}$ Universidade Federal de São Paulo, Escola Paulista de Medicina, Departamento de Neurologia e Neurocirurgia, São Paulo SP, Brasil.

Correspondence: Roberta Monterazzo Cysneiros; Rua da Consolação, 930 / Prédio 28;01302-907.São Paulo SP, Brasil. E-mail: rcysneiros@yahoo.com Conflict of interest: There is no conflict of interes to declare.

Supported: This work was sponsored by grants from Conselho Nacional de Desenvolvimento Científico e Tecnológico (CNPq-302528/2011-3) and Fundação de Amparo à Pesquisa do Estado de São Paulo (FAPESP-2011/50680-2). Fabiane Ochai Ramos was fellow from CNPq.

Received 11 November 2015; Received in final form 16 February 2016; Accepted 15 March 2016.
} 


\section{METHOD}

All procedures were approved by Universidade Presbiteriana Mackenzie Ethical Committee (CEUA 093/08/2012). Male Wistar rats were maintained under controlled conditions (07:00-19:00 hours, light/dark cycle; $22-24^{\circ} \mathrm{C}$ ).

\section{SE induction}

At 25 days of age the experimental animals were submitted to pilocarpine-induced epilepsy ${ }^{13}$. Experimental group (15 animals) received methyl scopolamine (1 mg/kg, s.c.) 30 minutes before pilocarpine administration (350 mg/kg, ip) and control group (11 animals) received saline $0.9 \%$. Three hours after the onset of the status epilepticus both groups received diazepam (7.5 mg/kg, sc) (Figure 1). Epilepsy induced experimentally exhibits high and variable mortality rates, and thus, the following groups of animals were designed:

Experimental group: 6 animals; Control group: 11 animals

The animals were monitored $24 \mathrm{~h}$ /day starting at the SE induction up to end of experiments. The behavioural tests (BT) started from 60 days postnatal after all animals have been evolved to epilepsy.

\section{Behavioural Tests (BT)}

The animals were transferred to the testing room 60 min before each day session. All apparatus were cleaned with a $5 \%$ alcohol solution after each behavioral procedure. At the end of behavioral tests, the rats were anaesthetized with urethane $1,200 \mathrm{mg} / \mathrm{kg}$ (ip). The half animals of each group were decapitated, the brains were dissected and frozen to $-80 \mathrm{C}$ and the other half was submitted to transcardial perfusion.

\section{Open field}

The apparatus consisted of a circular arena $(100 \mathrm{~cm}$ diameter) enclosed by plain white walls and a floor divided into 12 zones, being 8 peripheral and 4 central (Insight Ltda, Brazil). Each animal was placed into the central area and observed for $10 \mathrm{~min}$. During this time, the locomotor activity was expressed as the number of peripheral, central or total lines crossed. In addition, the time spent on central zone was measured. The test was repeated 7 and 15 days later.

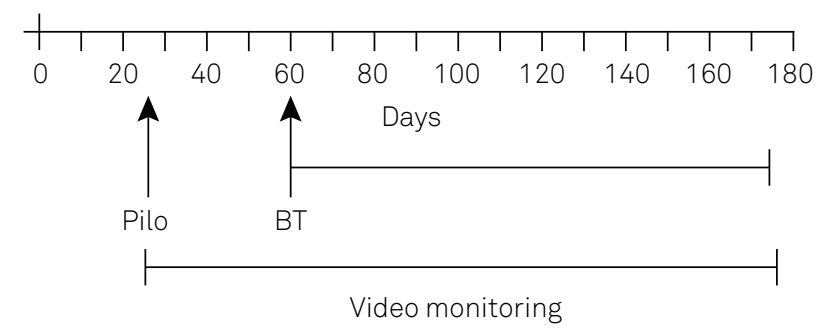

Figure 1. Timeline of the procedures. Pilo: picocalpine; BT: behavioural test.

\section{Elevated plus maze (EPM)}

The apparatus had two closed arms with walls $45 \mathrm{~cm}$ in height and two open arms $50 \mathrm{~cm}$ long (Insight Ltda, Brazil) and was elevated $50 \mathrm{~cm}$ from the floor. The animals were placed in the central zone of the maze with their nose pointing towards an open arm and explored the maze for 5 minutes. The number of entries and the time spent in both arms were recorded and expressed as percent of entries (EOA) or time (TOA) in open arms: ([Open arm /(Open arm + Closed arm) $]$ * 100). The test was repeated 7 and 15 days later.

\section{Operant conditioning box (OCB)}

The OCB aimed to analyse attention, impulsivity and visuospatial working memory in a visual discrimination task. The apparatus (Habitest Coulbourn Instruments) consisted of two responder levers, two cue lights located above the levers and a water dispenser. The apparatus' activities were controlled by Graphic State and the data stored to posterior analysis. The apparatus was enclosed in a sound-attenuating box outfitted with an exhaust fans.

The animals were evaluated using three experimental protocols, each one with fifteen sessions and lasting thirty minutes. Before that, the animals were habituated to the apparatus followed by acquisition sessions. The animals were deprived of water 21 hours before the procedures. For habituation, the animals remained 30 minutes in the apparatus; the reinforcement was not delivered and the cue lights above the levers were off. After that, the animals were subject for two acquisitions sessions, lasting 30 and $15 \mathrm{~min}$, respectively. The cue light located inside the apparatus was on, but the cue lights above the levers were not lit. The reinforcement was delivered every $10 \mathrm{~s}$ independently of the rat's behavior. During subsequent sessions, the learning was acquired by the method of successive approximations. During the initial sessions, the rats learned to press the left lever in order to receive reinforcement immediately after every correct response. The cue light above the left lever was now lit the entire session and the lights above the right lever were off. It was considered that the animals learned to press the left lever when it was pressed at least eight consecutively times in order to obtain the reinforcement. After that, the animals were trained to press the right lever following the same shaping procedure.

In the first and second protocols the light above the levers stayed lit for 5 or 1s, respectively, shifted randomly and the reinforcement was delivery immediately after the correct lever being pressed. In the third protocol, the light stayed lit for $1 \mathrm{~s}$, but the reinforcement was delivery after $10 \mathrm{~s}$. It was analysed the number lever presses and the percentage of correct responses. Correct responses were considered when the animal pressed the lever while the light points were lit. 


\section{Statistical analyses}

The data were expressed as mean \pm standard error and analysed by Mixed ANOVA, using Bonferroni for post-hoc testing. p-values of 0.05 or less were considered significant. The analyses were effectuated using commercial program (Prism 5.03 for windows).

\section{RESULTS}

\section{Frequency of seizures}

The first spontaneous seizure started between 4-15 days after SE onset with an average frequency of $1.34 \pm 0.27$ per week. Most seizures were recorded during the day light (67.74\%).

\section{Open field}

For peripheral locomotion, it was noted a significant difference between groups $\left(\mathrm{F}_{230}=5.365, \mathrm{p}=0.035\right)$, with no difference between sessions $\left(\mathrm{F}_{2,30}=0.167, \mathrm{p}=0.84\right)$ nor effect of interaction between factors $\left(\mathrm{F}_{2,30}=0.634, \mathrm{p}=0.53\right)$. The peripheral locomotion was higher in experimental group as compared to control (Figure 2A).

Central locomotion was a significantly different between groups $\left(\mathrm{F}_{2,30}=6.43, \mathrm{p}=0.022\right)$ and the sessions $\left(\mathrm{F}_{2,30}=3.81\right.$, $p=0.033$ ), with no effect of interaction between the factors $\left(F_{2,30}=0.20, p=0.81\right)$. The experimental group showed higher locomotor activity in the central zone as compared to control (Figure 2B).

The total locomotion was significantly higher in the experimental group as compared to control $\left(\mathrm{F}_{1,30}=6.24, \mathrm{p}=0.02\right)$, with no effect of interaction between the factors $\left(\mathrm{F}_{2,30}=0.64\right.$, $\mathrm{p}=0.52)$. Only control group exhibited reduction on locomotor activity over time (Figure 2C).

The time spent in the central zone was significantly different between groups $\left(\mathrm{F}_{1,30}=6.032, \mathrm{p}=0.026\right)$, with no effect of sessions $\left(\mathrm{F}_{2,30}=2.23, \mathrm{p}=0.12\right)$ nor interaction between the factors $\left(\mathrm{F}_{2,30}=0.94, \mathrm{p}=0.39\right)$. The time in the central zone was higher in the experimental group as compared to control (Figure 2D).

In a non-aversive context, animals with ELT exhibited hyperactivity and reduced level of anxiety-related behaviour.

\section{Elevated plus maze}

Total number of entries was significantly different between groups $\left(\mathrm{F}_{1,30}=5.25, \mathrm{p}=0.036\right)$, with no difference between sessions $\left(\mathrm{F}_{2,30}=1.29, \mathrm{p}=0.28\right)$ nor effect of interaction between factors $\left(\mathrm{F}_{2,30}=0.91, \mathrm{p}=0.41\right)$. The locomotor activity was higher in the experimental group as compared to control group (Figure $3 \mathrm{~A}$ ).

For the percentage of entries and the time spent on the open arms no differences was noted for groups $\left(\mathrm{F}_{1,30}=1.12\right.$, $\mathrm{p}=0.30 ; \mathrm{F}_{1,30}=0.27, \mathrm{p}=0.60$, respectively), neither for sessions $\left(F_{2.30}=1.67, p=0.20 ; F_{2.30}=0.14, p=0.86\right.$, respectively $)$, nor for interaction between factors $\left(\mathrm{F}_{2,30}=0.87, \mathrm{p}=0.42 ; \mathrm{F}_{2.30}=0.05\right.$, $p=0.94$, respectively (Figure 3B and 3C).

\section{Operant conditioning box}

Both groups learned to press the levers in the first session. In the following sessions, both bars were used to obtain the reinforcement. Cue lights located above the levers indicated which bar should be pressed. The cue lights light up randomly and remained lit for periods of time varying in accordance with the protocol (5 or 1 second).
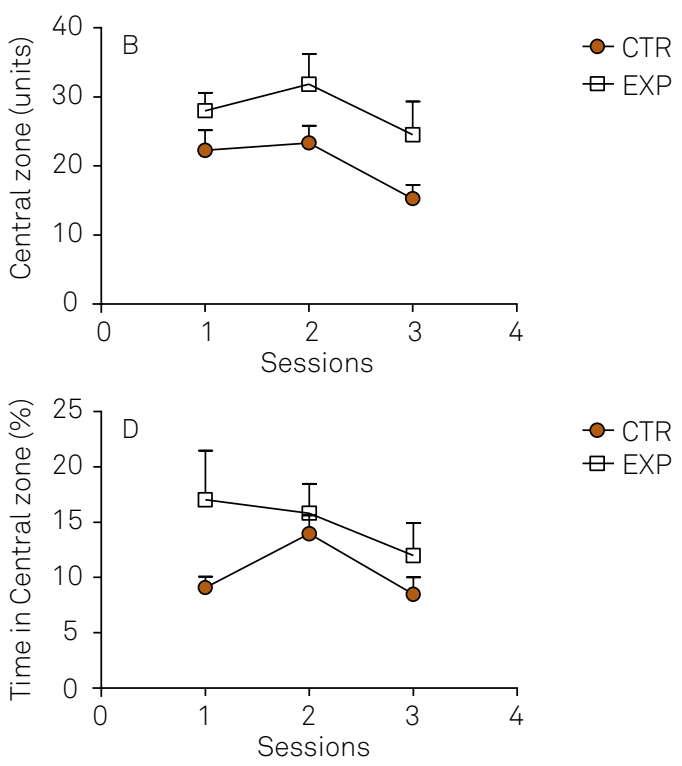

Figure 2. Peripheral (A), central (B), total locomotion (C) and time spent in central zone (D) on the open field in 3 sessions with 7 days apart were expressed as mean \pm standard error of CTR group $(n=11)$ and EXP group $(n=6)$. The experimental animals exhibited higher total and central locomotor activities and time spent in the central zone as compared to control, suggesting the presence of hyperactivity and reduced anxiety-related behavior. CTR: control; EXP: experimental. 


\section{Protocol 1}

It was analysed the percentage of the correct response and the number of bar presses. The percentage of correct responses was significantly different between session $\left(F_{14,210}=36.75, p<0.0001\right)$, with effect of interaction between factors $\left(\mathrm{F}_{1421}=3.57, \mathrm{p}<0.0001\right)$ and no difference between groups $\left(\mathrm{F}_{1,210}=0.379, \mathrm{p}=0.54\right.$, Figure $\left.4 \mathrm{~A}\right)$. Both groups improved their performance over time.

The number of the lever presses was significantly different between session $\left(\mathrm{F}_{14,21}=7.79, \mathrm{p}<0.0001\right)$, with no difference between groups $\left(\mathrm{F}_{[1,210]}=0.49, \mathrm{p}=0.07\right)$ and nor interaction between factors $\left(\mathrm{F}_{14,210}=1.63, \mathrm{p}=0.07\right.$, Figure $\left.4 \mathrm{~B}\right)$. The number of lever presses reduced to almost half concomitantly with the increase of the percentage of correct response which stabilized approximately in the twelfth session around of $60 \%$. The data suggests that the attention to the task was similar between groups.

\section{Protocol 2}

In order to increase the degree of difficulty in the subsequent 15 sessions, the cue lights remained lit for 1 second. The percentage of correct responses was significantly different across the sessions $\left(\mathrm{F}_{14,210}=3.32, \mathrm{p}<0.0001\right)$, with no effect of interaction $\left(\mathrm{F}_{14,210}=1.28, \mathrm{p}=0.21\right)$ nor difference between groups $\left(\mathrm{F}_{1,210}=3.43, \mathrm{p}=0.083\right.$, Figure $\left.5 \mathrm{~A}\right)$.
The number of lever presses was significantly different across the sessions $\left(\mathrm{F}_{14,210}=4.02, \mathrm{p}<0.0001\right)$, with no difference between groups $\left(\mathrm{F}_{1,210}=0.14, \mathrm{p}=0.71\right)$ nor interaction between factors $\left(\mathrm{F}_{14.210}=1.53, \mathrm{p}=0.099\right.$, Figure $\left.5 \mathrm{~B}\right)$.

\section{Protocol 3}

In order to assay the delay aversion and WM, the luminous spots remained lit for 1 second and the reinforcement was released 10 seconds after the correct lever being pressed.

For the percentage of correct response, it was noted a significant effect of interaction between factors $\left(\mathrm{F}_{14,210}=3.12, \mathrm{p}=0.0002\right)$ and effect of session $\left(\mathrm{F}_{14,210}=5.77\right.$, $\mathrm{p}<0.0001)$ with no difference between groups $\left(\mathrm{F}_{1,210}=4.39\right.$, $p=0.053$, Figure $6 \mathrm{~A}$ ). The percentage of correct response, in the initial sessions, was similar between groups, followed by improvement in control group and a moderate reduction in experimental group. In the 5th and the 12th session the percentage of correct response in the experimental group was significantly lower as compared to control group $(t=3.29, p<0.05$ and $t=3.15, p<0.05$, respectively). The number of lever presses was significantly different over time $\left(\mathrm{F}_{14210}=7.44, \mathrm{p}<0.0001\right)$ with significant effect of interaction between factors $\left(\mathrm{F}_{14.210}=2.62\right.$, $\mathrm{p}$ $<0.0016)$, with no difference between groups $\left(\mathrm{F}_{1,210}=0.73\right.$,
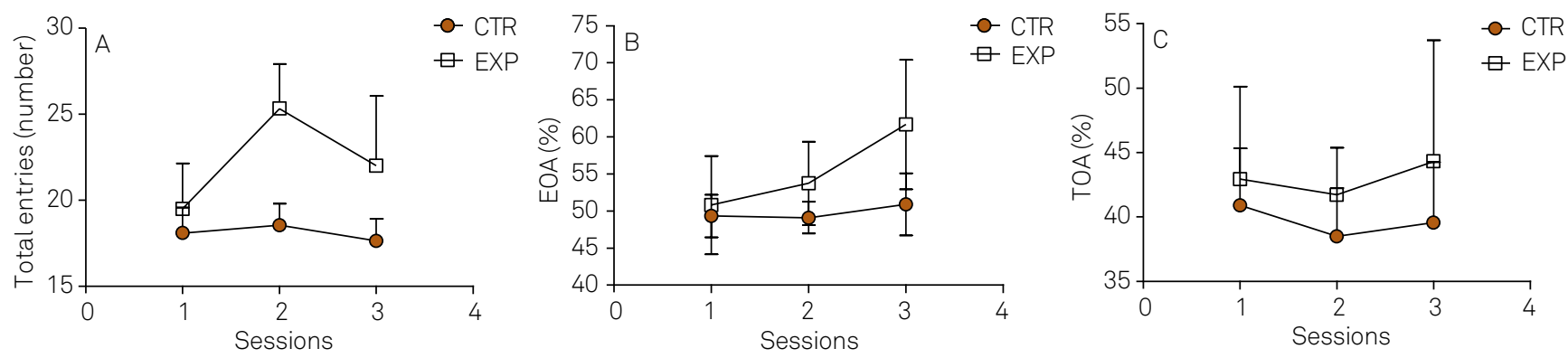

Figure 3. Total entries (A), percentage of entries in open arms (EOA\%, B), percentage of time in open arms (TOA \%, C) on the elevated plus maze in 3 sessions with 7 days apart were expressed as mean \pm standard error of the CTR group ( $n=11)$ and EXP group $(n=6)$. The experimental group exhibited higher locomotor activity as compared to control with no differences between groups for the percentage of entries, nor time spent in open arms. In an aversive context, the results showed that animals with epilepsy exhibited hyperactivity. CTR: control; EXP: experimental.
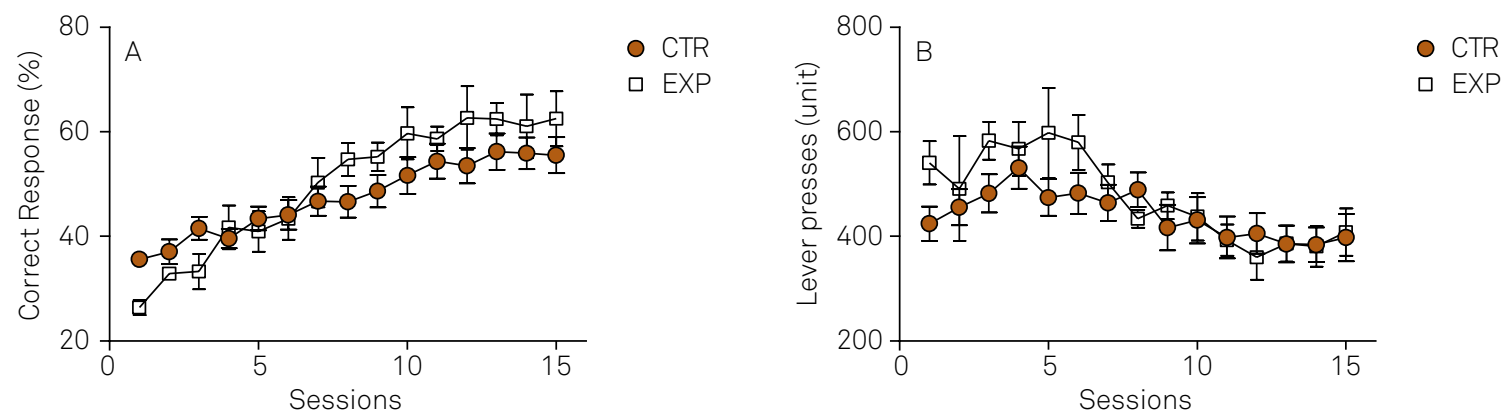

Figure 4. Operant Conditioning Box. The light above the levers stayed lit for $5 \mathrm{~s}$, shifted randomly and the reinforcement was delivery immediately after the correct lever being pressed. Percentage of correct answers (A) and total lever presses (B) expressed as mean \pm standard error of CTR $(n=11)$ and EXP $(n=6)$ groups. No significant differences were found between the groups. CTR: control; EXP: experimental. 
$p=0.40$, Figure $6 B$ ). The number of presses gradually decreased in both groups over time, stabilizing in a higher level in the experimental group.

The results of the three protocols were plotted on the same graph to better visualization and comparison the performance of both groups. When the difficulty of the challenge increases, the percentage of correct responses decreased. In the third protocol, note a sudden reduction in the percentage of correct response in both groups as compared to protocol 2, followed by recovery of the control group. For the experimental group, the percentage of correct response stabilized in a lower level as compared to previous protocol, and also when compared to control group within the same protocol (Figure 7A). Regarding the number of lever presses, note a drastic reduction in both groups when compared to the previous protocol. For the control group, the number of bar presses remained reduced with a concomitant increase in the percentage of correct response. For the experimental group, the number of lever presses stabilized in a higher level as compared to control, while the percentage of response stabilized in a lower level (Figure 7B).
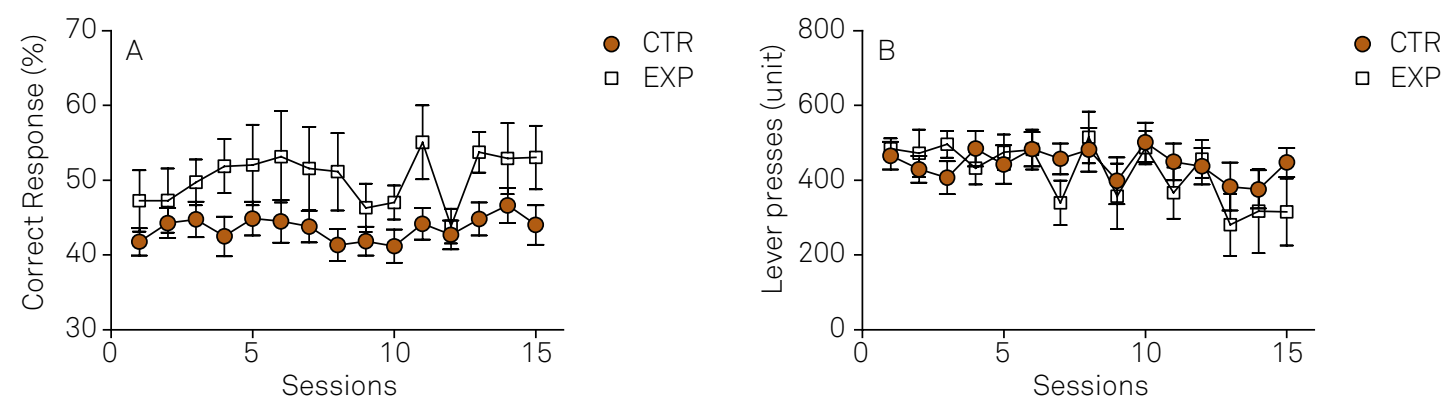

Figure 5. Operant Conditioning Box. The light above the levers stayed lit for $1 \mathrm{~s}$, shifted randomly and the reinforcement was delivery immediately after the correct lever being pressed. Percentage of correct answers (A) and total lever press (B) expressed as mean \pm standard error of CTR $(n=11)$ and EXP $(n=6)$ group. No significant differences were found between the groups. CTR: control; EXP: experimental.
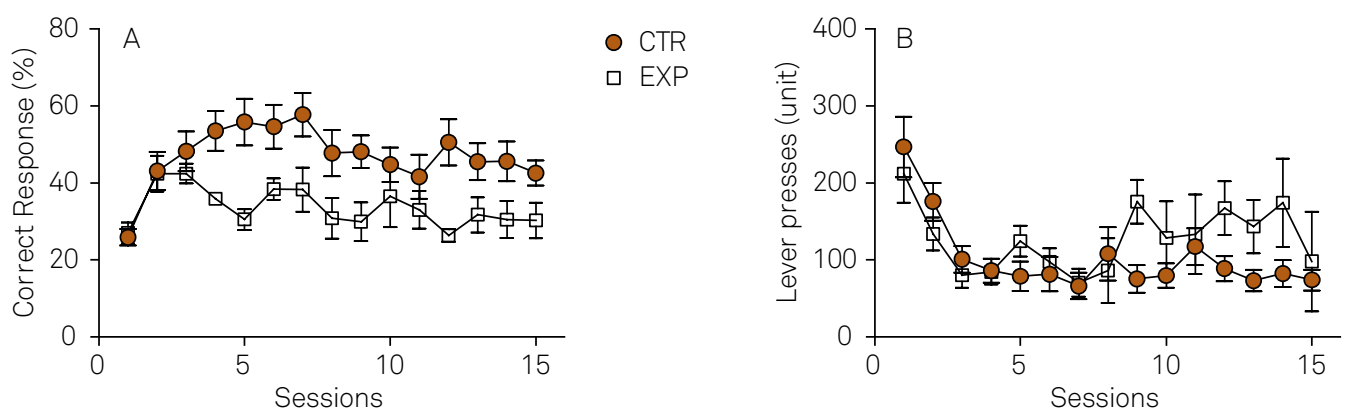

Figure 6. Operant Conditioning Box.The light above the levers stayed lit for $1 \mathrm{~s}$, shifted randomly and the reinforcement was delivery $10 \mathrm{~s}$ after the correct lever being pressed.Percentage of correct answers (A) and lever presses (B) expressed as mean \pm standard error of the CTR $(n=11)$ and EXP $(n=6)$ groups. The percentage of correct answers stabilized at a lower level and the lever presses stabilized at a higher level in the experimental group compared to the control group. CTR: control; EXP: experimental.
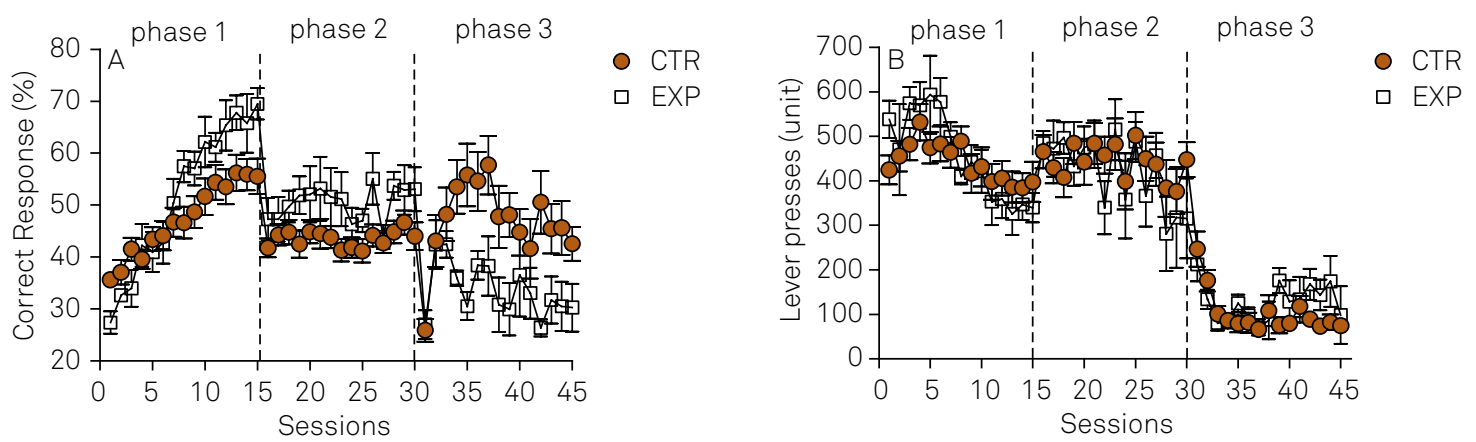

Figure 7. Operant Conditioning Box. Data of the three protocols were represented in the same graph. Percentage of correct answers (A) and number of lever presses (B). CTR: control; EXP: experimental. 
The results observed in protocols 1 and 2 suggest that animals with epilepsy showed no attention deficit in a visual discrimination task. In the protocol with the highest degree of difficulty, the difference between groups became evident. The performance of experimental animals reduced as compared to control, as result of the lower percentage of correct response and greater number of lever presses.

\section{DISCUSSION}

We investigated the presence of cognitive and behavioral dysfunctions in experimental TLE suggestive of concomitant presence of ADHD. Animals showed reduced anxiety-related behavior, hyperactive behavior, impulsivity and deficit in visuospatial working memory. Hyperactivity was observed over time in a familiar environment with neutral context (open field) and in a threatening environment. It is interesting to mention that the locomotor hyperactivity in children with ADHD as well as animal model of ADHD (e.g spontaneous hypertensive rats) tend to be less pronounced in novel environments than in familiar ones $^{14}$, and for that reason the test was repeated 3 times with 7 days apart. In addition, our results are in agreement with Kubova et al. ${ }^{15}$, which reported increased locomotor activity in rats submitted to kainic acid (KA) model at PN25 (25 day of life).

In a no aversive environment, experimental group exhibited reduced anxiety-related behavior with no changes in an aversive environment, suggesting that the state of anxiety is context dependent. In the lithium-pilocarpine (LIP) model, Detour et al. ${ }^{16}$ found increased number of entries and more time spent into the open arms of the aversive environment. Using the same paradigm, we did not observe difference between the groups, but a trend towards to increased activity in the open arms. The small sample size may have contributed to the discrepant results between studies. Inostroza et al. ${ }^{17}$ compared the cognitive and behavioral performance and anatomic changes between LIP model and KA model animals, which differed significantly in the pattern and extent of TLE-associated brain lesions. LIP-treated rats showed reduced state of anxiety against a slight decrease in the KA-treated rats. LIP-treated animals also exhibited increased motivation to consume sucrose, and both showed reduced motivation for social contact, being particularly affected LIP model.

After all behavioral tests, plasma corticosterone levels were increased only in LIP model, suggesting that altered emotional behaviors were not related to the epileptic condition; instead of probably reflect deregulation, model-dependent of the HPA axis. To the best of our knowledge, no study compared cognition, behavioral performance, anatomic changes and the pattern of seizure frequency between LIP model and pilocarpine model.
The OCB 's results suggest that learning, attention, and associative memory in a visual discrimination task were preserved in animals with epilepsy. However, when a delay for reinforcement was used, the performance was reduced, with increased impulsivity and visuospatial working memory impairment, which is prominent in ADHD. Interestingly, Pineda et al. ${ }^{18}$ observed in the LIP model that half the animals exhibited increased impulsivity and diminished attention in the lateralized reaction time test and the other half exhibited depressive related behavior. The seizure frequency ranging from 1 to 5 per week, but any correlation was investigated between the seizures frequency and behavioral changes. Faure et al. ${ }^{19}$ demonstrated in LIP model that animals displayed attention deficit with a tendency toward impulsivity and compulsivity in a five-choice serial reaction time task. The seizures frequency was not reported. In our study, animals did not display attention deficit, rather than, they exhibited increased impulsivity and visuospatial working memory impairment. Some issues may be underlying the differences in the animals' performance among studies.

An important issue to be considered regards the seizure frequency and its impact on the cognitive and behavioral changes. As seen in human condition, seizure activity is highly variable among animals and occurs in clusters with seizures free-intervals ${ }^{20,21}$. Bajorat et al. ${ }^{21}$ using video- $E E G$ in pilocarpine model identified three patterns of seizure distribution during the course of recording: a) > 3/day and evenly distributed seizures, b) $>2$ /day and again evenly distributed seizures and c) > 3/day and seizures clustered with seizure-free intervals. In our study, the average of seizures was 1.34 per week, being lower than observed by Bajorat et al. ${ }^{21}$. We do not rule that the low accuracy of the video monitoring may have contributed to underestimate the seizure activity. Despite of the well-established concept that epilepsy is a progressive disease and that the hippocampal/neocortical atrophy increases over time $\mathrm{e}^{22,23,24}$, remains the controversy if seizure frequency has substantial impact on brain damage and cognition. Fuerst et al. ${ }^{24}$ and Briellmann et al. ${ }^{25}$ showed a correlation between the seizures frequency and ipsilateral hippocampal volume loss. On the contrary, Liu et al. ${ }^{26}$ did not reported that the brain volume reduction was likely to be related to an initial brain insult and being influenced by age. Pacagnella et al. ${ }^{27}$ reported that the memory performance and the degree of hippocampal atrophy did not differ between patients with frequent and infrequent seizures, rather than, a positive correlation was found between age of onset and degree of hippocampal atrophy. In this sense, we argue that the lack of attentional deficits in our experimental animals could be related to the low seizure frequency and or the newly onset of epilepsy.

Another issue regards the degree of challenge of the task. The 5-choice serial reaction time task used by Faure et al. ${ }^{19}$ is more complex than the task used in our study. We used two 
lever presses and a stimulus presentation of $1 \mathrm{~s}$ or $5 \mathrm{sec}$. In the Faure and colleagues' study, five holes were used with a stimulus presentation of $0.5 \mathrm{~s}$ or $5 \mathrm{sec}$. With a stimulus presentation of $0.5 \mathrm{~s}$, which require a high attentional demand, the percentage of correct response was significantly different between groups, but not when the stimulus presentation was set at $5 \mathrm{sec}$. We argue that our protocol condition was not enough sensitive to detect attentional deficits.
In regarding the visuospatial working memory, experimental ${ }^{15}$ and clinical evidences ${ }^{9,28,29,30,31}$ have been shown that it is impaired in TLE. We showed evidences that animals with TLE exhibited hyperactivity, reduced level of anxiety-related behavior, increased mild impulsivity and impaired visuospatial working memory, suggesting that the pilocarpine model of epilepsy is appropriate to investigate the interplay between epilepsy and ADHD.

\section{References}

1. Sander JW. The epidemiology of epilepsy revisited. Curr Opin Neurol. 2003;16(2):165-70. doi:10.1097/00019052-200304000-00008

2. Boer HM, Mula M, Sander JW. The global burden and stigma of epilepsy. Epilepsy Behavior. 2008;12(4):540-6. doi:10.1016/j.yebeh.2007.12.019

3. Thurman DJ, Beghi E, Begley CE, Berg AT, Buchhalter JR, Ding D. Standards for epidemiologic studies and surveillance of epilepsy. Epilepsia. 2011;52(suppl 7):2-26. doi:10.1111/j.1528-1167.2011.03121.x

4. Laxer KD, Trinka E, Hirsch LJ, Cendes F, Langfitt J, Delanty N et al. The consequences of refractory epilepsy and its treatment. Epilepsy Behav. 2014;37:59-70. doi:10.1016/j.yebeh.2014.05.031

5. Duncan JS, Sander JW, Sisodiya SM, Walker MC. Adult epilepsy. Lancet. 2006;367(9516):14. doi:10.1016/S0140-6736(06)68477-8

6. Kwan P, Sander JW. The natural history of epilepsy: an epidemiological view.J Neurol Neurosurg Psychiatry. 2004;75(10):1376-81. doi:10.1136/jnnp.2004.045690

7. Sander JW. Some aspects of prognosis in the epilepsies: a review. Epilepsia. 1993;34(6):1007-16. doi:10.1111/j.1528-1157.1993.tb02126.x

8. Zamarian L, Trinka E, Bonatti E, Kuchukhidze G, Bodner T, Benke T et al. Executive functions in chronic mesial temporal lobe epilepsy. Epilepsy Res Treat. 2011;2011:ID596174. doi:10.1155/2011/596174

9. Stretton J, Winston GP, Sidhu M, Bonelli S, Centeno M, Vollmar C et al. Disrupted segregation of working memory networks in temporal lobe epilepsy. Neuroimage Clin. 2013;2:273-81. doi:10.1016/j.nicl.2013.01.009

10. Cohen R, Senecky Y, Shuper A, Inbar D, Chodick G, Shalev V et al. Prevalence of epilepsy and attention-deficit hyperactivity (ADHD) disorder: a population-based study.J Child Neurol. 2013;28(1):120-3. doi:10.1177/0883073812440327

11. Dunn DW, Austin JK, Harezlak J, Ambrosius WT. ADHD and epilepsy in childhood. Develop Med Child Neurol. 2003;45(1):50-4. doi:10.1111/j.1469-8749.2003.tb00859.x

12. Loutfi KS, Carvalho AM. Possiveis interfaces entre TDAH e epilepsia. J Bras Psiquiatr. 2010;59(2):146-55. doi:10.1590/S0047-20852010000200011

13. Turski WA, Cavalheiro EA, Schwarz M, Czuczwar SJ, Kleinrok Z, Turski L. Limbic seizures produced by pilocarpine in rats: behavioural, electroencephalographic and neuropathological study. Behav Brain Res. 1983;9(3):315-35. doi:10.1016/0166-4328(83)90136-5

14. Levant B, Zarcone TJ, Davis PF, Ozias MK, Fowler SC. Differences in methylphenidate dose response between periadolescent and adult rats in the familiar arena-novel alcove task.J Pharmacol Exp Ther. 2011;337(1):83-91. doi:10.1124/jpet.110.174425

15. Kubová H, Haugvicová R, Suchomelová L, Mares P. Does status epilepticus influence the motor development of immature rats? Epilepsia. 2000;41 (Suppl 6):S64-9. doi:10.1111/j.1528-1157.2000.tb01559.x
16. Detour J, Schroeder H, Desor D, Nehlig A. A 5-month period of epilepsy impairs spatial memory, decreases anxiety, but spares object recognition in the lithium-pilocarpine model in adult rats. Epilepsia. 2005;46(4):499-508. doi:10.1111/j.0013-9580.2005.38704.x

17. Inostroza M, Cid E, Menendez de la Prida L, Sandi C. Different emotional disturbances in two experimental models of temporal lobe epilepsy in rats. PLoS One. 2012;7(6):e38959. doi:10.1371/journal.pone.0038959

18. Pineda E, Jentsch JD, Shin D, Griesbach G, Sankar R, Mazarati A. Behavioral impairments in rats with chronic epilepsy suggest comorbidity between epilepsy and attention deficit/hyperactivity disorder. Epilepsy Behav. 2014;31:267-75. doi:10.1016/j.yebeh.2013.10.004

19. Faure JB, Marques-Carneiro JE, Akimana G, Cosquer B, Ferrandon A, Herbeaux K et al. Attention and executive functions in a rat model of chronic epilepsy. Epilepsia. 2014;55(5):644-53. doi:10.1111/epi.12549

20. Arida RM, Scorza FA, Peres CA, Cavalheiro EA. The course of untreated seizures in the pilocarpine model of epilepsy. Epilepsy Res. 1999;34(2-3):99-107. doi:10.1016/S0920-1211(98)00092-8

21. Bajorat R, Wilde M, Sellmann T, Kirschstein T, Köhling R. Seizure frequency in pilocarpine-treated rats is independent of circadian rhythm. Epilepsia. 2011;52(9):e118-22. doi:10.1111/j.1528-1167.2011.03200.x

22. Liu RS, Lemieux L, Bell GS, Hammers A, Sisodiya SM, Bartlett PA et al. Progressive neocortical damage in epilepsy. Ann Neurol. 2003;53(3):312-24. doi:10.1002/ana.10463

23. Cascino GD. Progressive damage in epilepsy. Epilepsy Curr. 2003;3(6):214-5. doi:10.1046/j.1535-7597.2003.03615.x

24. Fuerst D, Shah J, Shah A, Watson C. Hippocampal sclerosis is a progressive disorder: a longitudinal volumetric MRI study. Ann Neurol. 2003;53(3):413-6. doi:10.1002/ana.10509

25. Briellmann RS, Berkovic SF, Syngeniotis A, King MA, Jackson GD. Seizure-associated hippocampal volume loss: a longitudinal magnetic resonance study of temporal lobe epilepsy. Ann Neurol. 2002;51(5):641-4. doi:10.1002/ana.10171

26. Liu RS, Lemieux L, Bell GS, Sisodiya SM, Bartlett PA, Shorvon $S D$ et al. Cerebral damage in epilepsy: a population-based longitudinal quantitative MRI study. Epilepsia. 2005;46(9):1482-94. doi:10.1111/j.1528-1167.2005.51603.x

27. Pacagnella D, Lopes TM, Morita ME, Yasuda CL, Cappabianco FA, Bergo F et al. Memory impairment is not necessarily related to seizure frequency in mesial temporal lobe epilepsy with hippocampal sclerosis. Epilepsia. 2014;55(8):1197-204. doi:10.1111/epi.12691

28. Abrahams S, Morris RG, Polkey CE, Jarosz JM, Cox TC, Graves M et al. Hippocampal involvement in spatial and working memory: a structural MRI analysis of patients with unilateral mesial temporal lobe sclerosis. Brain Cogn. 1999;41(1):39-65. doi:10.1006/brcg.1999.1095 
29. Krauss GL, Summerfield M, Brandt J, Breiter S, Ruchkin D. Mesial temporal spikes interfere with working memory. Neurology. 1997;49(4):975-80. doi:10.1212/WNL.49.4.975

30. Owen AM, Morris RG, Sahakian BJ, Polkey CE, Robbins TW. Double dissociations of memory and executive functions in working memory tasks following frontal lobe excisions, temporal lobe excisions or amygdalo-hippocampectomy in man. Brain. 1996;119(5):1597-615. doi:10.1093/brain/119.5.1597

31. Doucet G, Osipowicz K, Sharan A, Sperling MR, Tracy JI. Hippocampal functional connectivity patterns during spatial working memory differ in right versus left temporal lobe epilepsy. Brain Connect. 2013;3(4):398-406. doi:10.1089/brain.2013.0158 